\title{
Drug-Herb Interaction between Metformin and Momordica charantia in Diabetic Mice
}

\author{
Asri Dwi Endah Dewi Pramesthi ${ }^{1}$, Mirhansyah Ardana², Niken Indriyanti ${ }^{3}$ \\ ${ }^{1}$ Research and Development FARMAKA TROPIS Laboratory, Faculty of Pharmacy, Universitas Mulawarman, Samarinda, \\ Indonesia \\ ${ }^{2}$ Department of Pharmaceutical and Technology, Faculty of Pharmacy, Universitas Mulawarman, Samarinda, Indonesia \\ ${ }^{3}$ Department of Pharmacology, Faculty of Pharmacy, Universitas Mulawarman, Samarinda, Indonesia
}

Background: Bitter gourd has various metabolites, such as momordicosides, polypeptide-P, $v$-insulin, charantin, and vicine that have antidiabetic effect. It has synergistically effect while combined with oral diabetic drugs, such as metformin as glucose lowering agent. The aim of this study is to investigate the interaction of bitter gourd fruit juice and metformin as glucose lowering agent in mice.

Materials and Methods: Alloxan-induced diabetic mice were treated with bitter gourd fruit juice, metformin, and the combination of those two for 21 days. Glucose level was checked on first and last day of treatment.

Results: Furthermore, blood glucose levels measurement showed no significant difference between groups compared with negative control, which was $p>0.05$. The stomach of groups that treated with metformin and bitter gourd fruit juice histopathologically showed no significant differences.

Conclusion: The use of bitter gourd once daily together with metformin is a better choice, while twice daily might induce hypoglycemia and mice death. There is no interaction between them on lowering blood glucose.

Keywords: metformin, Momordica charantia, diabetes mellitus

\section{Introduction}

Drug and food or drug and traditional medicine interaction that mostly happens in patients who receive antidiabetic therapy might impacting the effect of therapy. One of them is the interaction between metformin and bitter gourd fruit juice. Bitter gourd has so many metabolites such as vicine, charantin, glycosides and caravylosides. Saponins that

Date of submission: December 11, 2018

Last Revised: March 4, 2019

Accepted for publication: March 4, 2019

Corresponding Author:

Asri Dwi Endah Dewi Pramesthi

Research and Development FARMAKA TROPIS Laboratory

Faculty of Pharmacy, Universitas Mulawarman

Jl. Kuaro, Gunung Kelua, Samarinda, Indonesia

E-mail: pramesthiasri@gmail.com contained in bitter gourd, like steroid and triterpenoid have a good antidiabetic effect. ${ }^{1}$ Another example of its metabolites are momordicosides, momordicilin and momordenol. Momordicosides stimulate the translocation of glucose transporter 4 (GLUT4) to the cell membrane and enhance the uptake of glucose. Momordicilin and momordenol might block the active site of glycogen synthase kinase-3 (GSK3 ) and exhibit anti-diabetic activities. ${ }^{2}$ Metformin and bitter 
gourd mechanism interaction referred as synergistically interaction, because one of three mechanism of metformin acts as 5' AMP-activated protein kinase (AMPK)-signalling activator, increases insulin receptor sensitivity, and stimulates translocation of glucose via GLUT4. ${ }^{3}$ Small noncontrolled studies have subsequently shown that bitter gourd produces a significant improvement in glucose tolerance in patients with type 2 diabetes, both when they are taking metformin and when they are not taking antidiabetics. In these studies, bitter gourd was given orally as a juice from the fruit, dried powdered fruit, fried fruits, aqueous extract, or solvent extract from the fruit. ${ }^{4}$

Metformin is the first biguanides introduced in 1957 and was approved in 1994. The main mechanism of metformin is reduce hepatic glucose production by inhibiting gluconeogenesis. It also enhances peripheral insulin sensitivity in the skeletal muscle by increasing insulin receptor tyrosine kinase activity and GLUT-4 translocation to the cell membrane. And then, it could improve beta-cell responsiveness to a glucose load through correction of glucose toxicity. ${ }^{5}$

Based on the mechanism of metformin and bitter gourd, there was no study has yet been conducted to investigate the interaction between them. The present study was conducted to investigate the effect of metformin and bitter gourd fruit juice on blood glucose level in mice with alloxan-induced diabetes.

\section{Materials and methods}

\section{Extraction}

Bitter gourd was purchased from the local market at Sungai Kunjang, Samarinda, East Borneo, Indonesia. It was used with the prior approval of Forest Dendrology and Ecology Laboratory, Faculty of Forestry, Universitas Mulawarman (No. 27/UNI7.4.3.08/LL/2018). Twenty grams of bitter gourds were washed until cleaned thoroughly and the excess water was dab-dries with a towel before weighing. It was placed in a heat-resist container then was added by $100 \mathrm{~mL}$ aquadest, and cooked in a high power microwave for 5 minutes. The cooked bitter gourd and remaining liquid were cooled rapidly on the refrigerator to prevent further cooking from residual heat and then homogenized with a blender. The homogenate was filtered by strainer and the liquid was given to the mice. ${ }^{6}$ The juice was given based on mice's body weight and its stomach capacity, while the concentration was $77.58 \mathrm{~g} / \mathrm{mL}$.

\section{Laboratory Animals}

In this experimental study, 30 male DDY mice weighing 20$40 \mathrm{~g}$ were purchased from LPPT Gadjah Mada University, Jogjakarta, Indonesia, and maintained under appropriate laboratory conditions with free access to similar ad libitum and water. Mice were used with the prior approval of the Animal Ethics Committee, Faculty of Medicine, Universitas Mulawarman (No. 27/KEPK-FK/VII/2018).

\section{Diabetes Induction and Animal Grouping}

Thirty mice were randomly selected and 5 of them were separated as control group or normal group. The other mice were induced by Alloxan $180 \mathrm{mg} / \mathrm{kg}$ in $\mathrm{NaCl} 0.9 \%$, 7 times in 14 days. Before measuring the blood glucose, mice were fasted for about 2-3 hours. The blood glucose was measured by taking from using tail puncture method on the first day of induction, and then checked by using glucometer (Autocheck Multi Monitoring System 3 in 1 Meter, SN AH03011149). After induction and confirmation of diabetes, 25 mice were divided into 5 groups consist of 5 mice each group. Normal group, positive control group (metformin), negative control group, bitter gourd group, bitter gourd + metformin once daily group (MBG), and last group was bitter gourd + metformin twice daily group (MBG 2). All administrations were conducted by gavage and at a specific time of the day.

\section{Data Analysis}

The data distribution was measured using Shapiro-Wilk test in SPSS. All variables were met the normal distribution assumption in each group, so the ANOVA test was used to compare the variables between groups and Bonferroni and Games-Howell test was used for multiple comparisons. Statistical analysis was done by SPSS ver.22 with the significancy level $p<0.05$.

\section{Results}

In this investigation, the alloxan-induced diabetes in 25 mice. It was divided into five groups beside the normal group. Body weight of each group were fluctuated, where the highest body weight was measured in metformin and bitter gourd group, while the lowest one was seen in positive control groups that was treated with metformin. The body weight of each group seems fluctuated as seen in Figure 1. The negative control group showed the highest weight was on day 15 and the lowest one was in day 12. The bitter gourd 


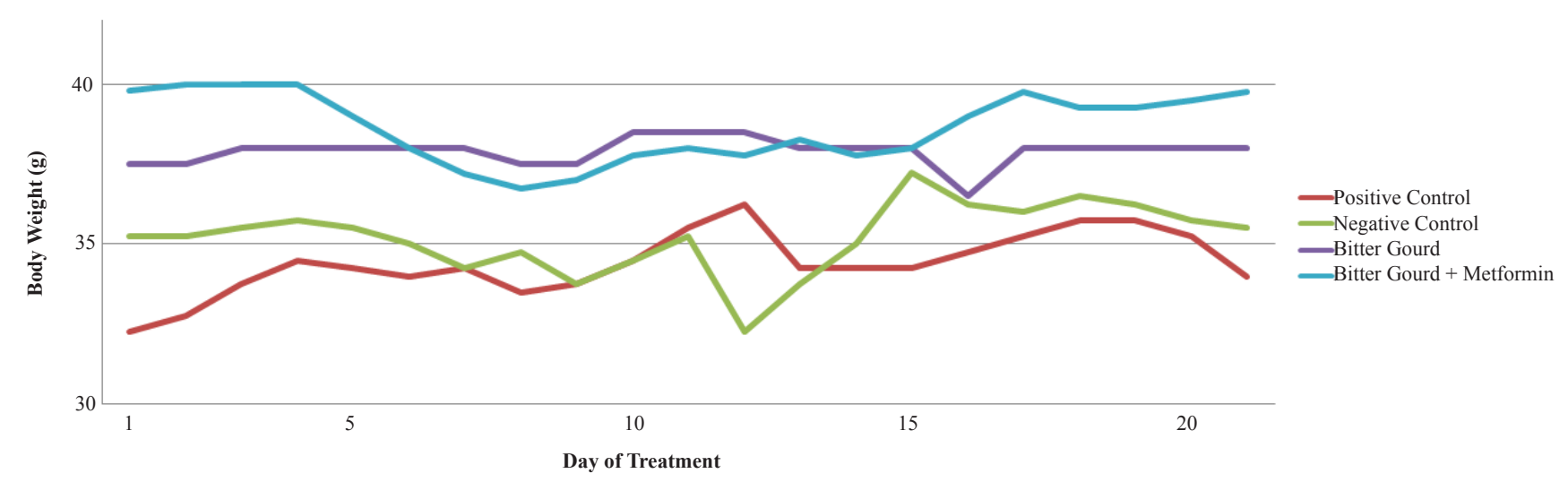

Figure 1. Average body weight of mice was measured in treatment days.

group showed almost static in body weight changed while treatment days.

Blood glucose level have decreased from before and after treatment. The highest to the lowest blood glucose level that was measured before the treatment was the negative control group, followed by bitter gourd group, MBG group, positive control group, then normal group. While after the treatment each group showed decreases in blood glucose levels as shown in Figure 2 and Table 1.

MBG 2 group, which was treated with metformin combined with bitter gourd twice daily could decrease blood glucose level significantly compared to other groups. Blood glucose level was measured before and 30 minutes after treatment were performed orally in day 4 . Mice number 1 , 2 , and 5 show a decrease in blood glucose level after 30 minutes. The result can be seen in Table 2 .

Compared to normal groups, each group organs had increased. Organ index number of spleen and stomach was increased significantly compared to other organs.
Significancy of organ indexes of stomach and spleen were both $p<0.05$. The number of increasing in organ index of mice's organs can be seen in Table 3.

Data was distributed normally after test of normality was performed with there were no significant differences between each group $(p>0.05)$ then ANOVA was performed. Since then negative control, positive control, bitter groud and MBG groups showed there were no significant differences $(p>0.05)$ between groups.

Histopathological observations show in Figure 3. In the negative control group's stomach was found two locations of large ulcer with corneum epithelium and keratin cell were thickened. It was also shown that there was much debris while vascularization congested but the form of the gastric pit was normal. Positive control that is treated with metformin, the black arrow shows the location of focal hyperplasia with epithelium proliferation. The green arrow shows the width of ulcer that formed after treatment. In the bitter gourd group, the inflammation of cell infiltration was

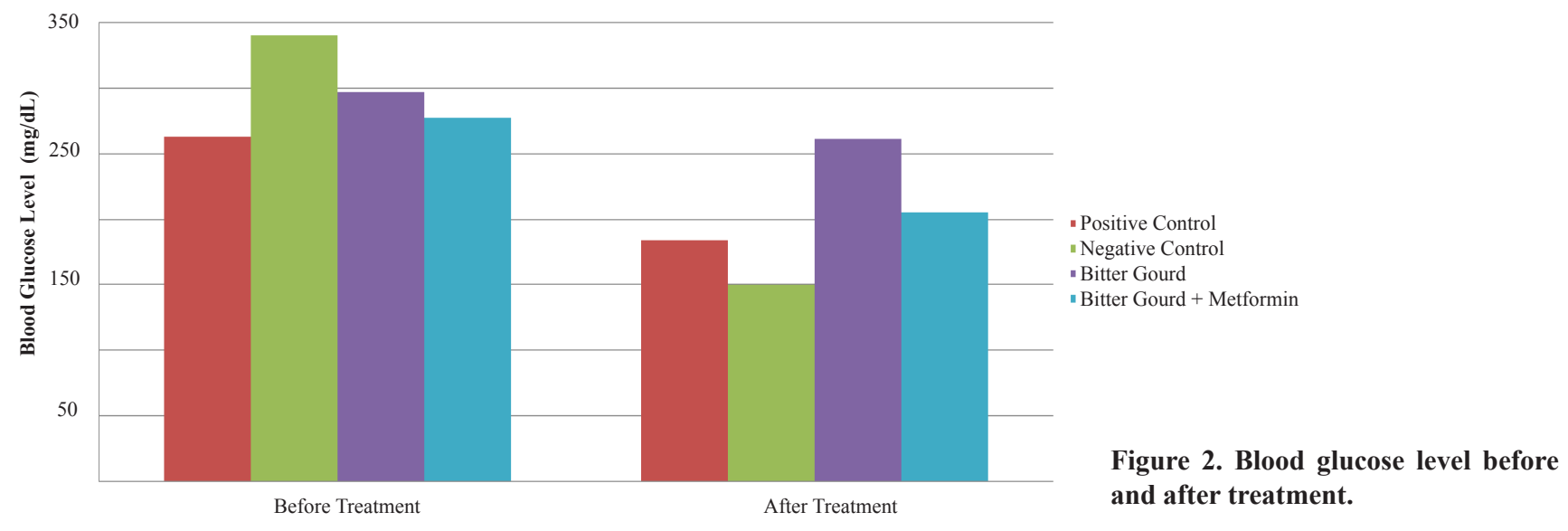


Table 1. Average \pm standard deviation of blood glucose level before and after treatment were performed.

\begin{tabular}{|c|c|c|}
\hline \multirow[t]{2}{*}{ Group } & \multicolumn{2}{|c|}{$\begin{array}{l}\text { Average } \pm \text { Standard Deviation of } \\
\text { Blood Glucose Level (mg/dL) }\end{array}$} \\
\hline & Before & After \\
\hline Control (+) & $263.3 \pm 94.87$ & $184.3 \pm 62.13$ \\
\hline Control (-) & $340 \pm 220.60$ & $150 \pm 36.72$ \\
\hline Bitter Gourd & $297 \pm 31.11$ & $261.5 \pm 60.10$ \\
\hline Bitter Gourd + Metformin & $277.75 \pm 113.73$ & $205.5 \pm 50.44$ \\
\hline
\end{tabular}

found, with one focal hyperplasia and small keratinization was formed. The gastric pit with complete severe erosion and increasing of parietal cell is occurred in MBG group. There was one focal hyperplasia shows with thickened keratinization. There are two locations of focal hyperplasia with hyperkeratosis and gastric pit form was considered to normal in the stomach of the normal group.

\section{Discussion}

Body weight of each group are fluctuated during treatment days. MBG group shows the highest average body weight in the first day of treatment and start decreasing in day 5 until 8. In day 9 to 21 was fluctuatedly increasing. Bitter groud group average body weight is under the MBG group. Which is fluctuated around $33-37 \mathrm{~g}$. Negative and positive control group are fluctuated slightly similar in body weight average number. It is between $32-36 \mathrm{~g}$.

Metformin is well-known as a treatment for obesity. It has been found effective to decrease adiposity and obesityassociated conditions in both human and animal. ${ }^{7}$ Metformin induces an increase in mitochondrial mass like leptin, which is a hormone increasing energy expenditure and suppressing appetite. ${ }^{8}$ Reducing the absorption of carbohydrates from the
Table 2. Blood glucose level of MBG 2 group before and after 30 minutes of treatment in treatments day 4 .

\begin{tabular}{ccc}
\hline \multirow{2}{*}{\begin{tabular}{c} 
MBG 2 \\
\cline { 2 - 3 }
\end{tabular}} & \multicolumn{2}{c}{ Blood Glucose Level (mg/dL) } \\
\cline { 2 - 3 } 1 & Before Treatment & After $\mathbf{3 0}$ Minutes \\
\hline 2 & 403 & 321 \\
3 & $\mathrm{Xi}$ & 573 \\
4 & 482 & 542 \\
5 & 156 & 156 \\
\hline
\end{tabular}

gut, suppressing hepatic glycogenesis and hepatic glucose-6 phosphate, and inhibiting hepatic gluconeogenesis are other actions of metformin that may beneficial for obesity. ${ }^{9}$ Bitter gourd has phenolic compound such as simple phenolic acids and flavonoids. It has attenuated in vitro adipogenesis by activating AMPK signal pathway in preadipocytes and decreasing expression of adipogenesis-related factors and hypolipidemic effect and lowers the risk of high fat dietinduced obesity and reduces serum cholesterol. ${ }^{10}$

Mechanism of both metformin and bitter gourd represent its ability to decreasing body weight in treatment days. Bioactive compounds of bitter gourd may infect the variance of body weight. Suppressing appetite is one of the side effects that may happen, so it can influence the fluctuated body weight. Body weight of each group were fluctuated during treatments day as seen in Figure 1.

In this study, the combination of bitter gourd and metformin as antidiabetic therapy might decrease blood glucose level significantly. Blood glucose level was measured before and after treatment shows that blood glucose level was decreased significantly for each group. The data can be seen in Table 1. At first, this study was consist of 6 groups.

Table 3. Average \pm standard deviation of organ indexes of each group after treated for 21 days.

\begin{tabular}{lcccc}
\hline \multirow{2}{*}{ Groups } & \multicolumn{4}{c}{ Average \pm Standar Deviation (\%) } \\
\cline { 2 - 5 } & Liver & Stomach & Spleen & Kidney \\
\hline Normal & $4.697 \pm 0.44$ & - & $0.360 \pm 0.09$ & $0.938 \pm 0.29$ \\
Control (+) & $5.721 \pm 1.43$ & $0.839 \pm 0.38$ & $0.803 \pm 0.33$ & $1.287 \pm 0.37$ \\
Control (-) & $5.637 \pm 0.66$ & $1.3675 \pm 0.27$ & $0.793 \pm 0.19$ & $1.408 \pm 0.25$ \\
Bitter Gourd & $5.164 \pm 0.06$ & $1.009 \pm 0.23$ & $0.67 \pm 0.04$ & $1.364 \pm 0.02$ \\
Bitter Gourd + Metformin & $5.665 \pm 0.54$ & $1.907 \pm 0.35$ & $0.897 \pm 0.19$ & $1.426 \pm 0.10$ \\
Significancy & $\mathbf{0 . 3 9 7}$ & $\mathbf{0 . 0 0 5}$ & $\mathbf{0 . 0 2 7}$ & $\mathbf{0 . 2 7 4}$ \\
\hline
\end{tabular}



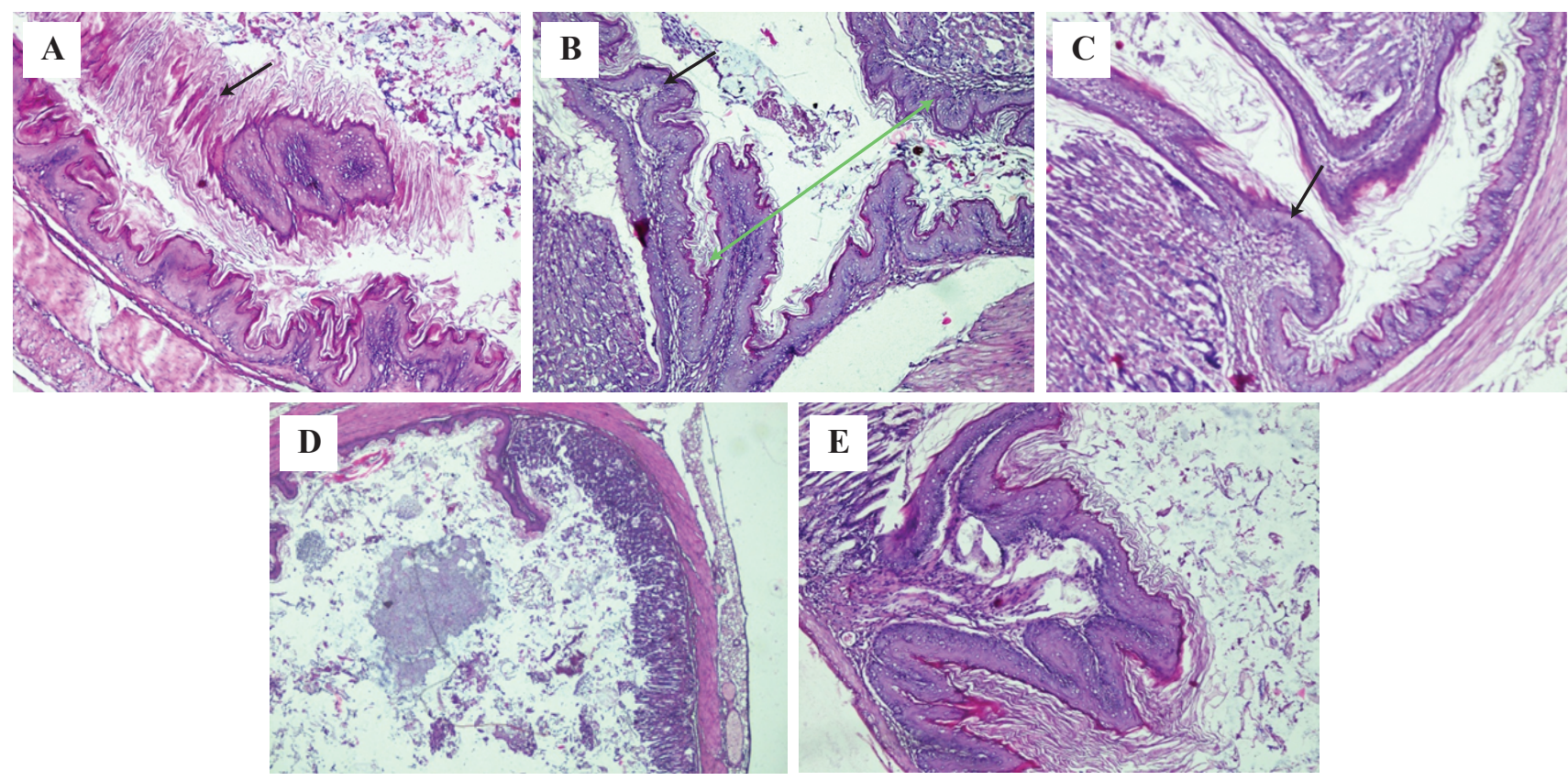

Figure 3. The stomach histology observation after treated for $\mathbf{2 1}$ days based on each group. A: Negative control group; B: Positive control; C: Bitter groud; D: Bitter groud + metformin; E: Normal; Black arrow: focal hyperplasia with epithelium proliferation; The green arrow: width of ulcer that formed after treatment. (Magnitude of 100x by utilizing of inverted microscope Olympus CKX410)

One of those group is MBG 2 or MBG that was given twice a day. In the first week, mice in those group was died one by one. It might be caused by the metabolites of bitter gourd have some interactions with metformin so the blood glucose level decreased significantly. Blood glucose level decreased in MBG 2 group significantly as seen in Table 2.

Bitter gourd (Momordica charantia) showed significant reduction of blood sugar level immediately and after 120 minutes of consuming bitter gourd juice in patients with type 2 diabetes mellitus. ${ }^{11}$ The consumption of $2.5 \mathrm{~g}$ dry bitter gourd (equivalent to $50 \mathrm{~g}$ fresh bitter gourd) may depict an effective approach to lower elevated blood glucose levels in individuals with prediabetes. The glucoselowering effect of bitter gourd was found higher among participants who started with higher baseline fasting plasma glucose levels. As the current study included prediabetic participants only, the glucose-lowering effect of bitter gourd is expected to be even more pronounced among patients with higher glucose levels. ${ }^{12}$ In diabetic streptozotocin-rats, the concentration of plasma insulin significantly increased at 4 and 6 hours in both normal and diabetic groups by using $10 \mathrm{mg} / \mathrm{kg}$ bitter gourd extract. ${ }^{1}$ Antidiabetics such as metformin, chlorpropamide, tolbutamide, glibenclamide and glinide, blood-glucose lowering effects can be increased by bitter gourd. Hypoglycaemic coma and seizures occurred in two young non-diabetic children after they were given bitter gourd tea. ${ }^{4}$

Based on the mechanism of metformin that has been used widely for over 50 years, it has been found to be safe and efficacious both as monotherapy and in combination with other antidiabetics therapy such as bitter gourd. Previous studies reported that bitter gourd had beneficial effects in reducing blood glucose level. Based on the blood glucose level that been measured, metformin, while combined with bitter gourd is safe for once daily intake. Bitter gourd seems to have a therapeutic effect on lowering blood glucose as effective as metformin does.

Organ index measurement was performed to support the blood glucose level data in mice. Liver, spleen, kidney, and stomach indexes were measured in this study. Compared to normal group, there were no significant differences organs swelling. Kidney indexes also shows no significant swollen. The therapy that was done in this study did not affect the liver and kidney of the mice. It can conclude that the combination of metformin and bitter gourd have no side effects to those organs. While the spleen of each group swells 2-3 times higher than the normal one. The spleens from each group show significantly difference $(p<0.05)$. 
The differences between groups show that stomachs are significantly different $(p<0.05)$.

Bitter gourd has polysaccharides possess immunomodulatory effects both in vitro and in vivo. Other study said that the immunity enhancement of bitter gourd polysaccharides has strong immune-enhancing activity and may improve the immune function by stimulating the activation of lymphocytes and macrophages. ${ }^{13}$ The mammalian spleen is a peripheral lymphoid organ that plays a central role in host defense. The spleen cells proliferated to produce the cytokines as an effort to block the immune reactivity. The $\mathrm{T}$ regulators are produced in this organ as immune homeostasis. ${ }^{14,15}$

Differences between each groups' spleen indicate that the immune-regulation imbalance resulted from the bitter groud effect is processed to be conquered by the spleen function. Another difference is seen in the stomach. The basic properties of bitter gourd's metabolites may affect gastric pit of mice. The clear differences between groups treatment also occurred in the stomach histology.

Metformin has side effects, notably gastrointestinal complaints..$^{16}$ The most frequent side effect of metformin are gastrointestinal disturbance, such as flatulence, abdominal pain and loss of appetite. ${ }^{8}$ Histopathological observation used to investigate side effect of metformin and bitter gourd. It is shown that negative control was found two locations of the wide ulcer with thickening of corneum epithelium and hyperkeratinization. It also shown much debris with vascularization look congested with hyperemia and gastric pit seems normal. In positive control that was treated with metformin shown that gastric pit seems normal, focal hyperplasia occurred with epithelium erosion up to stomach's lumens with cell debris. In bitter groud groups there was one location of smaller focal hyperplasia compared to the previous groups with keratinization and there were some debris and cell infiltration. MBG group shows the complete severe erosion that makes the gastric pit looks shorter and diffused. It also shows one hyperplasia with large keratinization. There is increasing in the parietal cell that makes increasing in $\mathrm{HCl}$ production. While in the normal group there were two locations of focal hyperplasia with hyperkeratosis and gastric pit form was considered to normal.

Glandular stomach lesions are quite rare in National Toxicology Program (NTP) mouse studies. Hyperplasia of the glandular stomach mucosa is not common in controls but may be seen in regenerative hyperplasias, such as at the margin of an ulcer erosion. Glandular hyperplasia is characterized by an increase in density of glands within the mucosa. In rats, hyperplasia may be a preneoplastic lesion. Focal hyperplasia is seen occasionally in mice. However, in mice, this is not considered to be a preneoplastic change. The incidence is not increased in studies with adenocarcinomas of the glandular stomach, and progression from focal hyperplasia to adenocarcinoma has not been observed. ${ }^{17}$

Bitter gourd has secondary metabolites that most of them have basic properties. The basic properties of bitter gourd may affect the gastric pit. Focal hyperplasia in normal mice is considered to be normal. MBG group shows the complete severe erosion caused by the properties of metformin and bitter gourd itself.

\section{Conclusion}

Metformin and bitter gourd consumed once daily is considered to be safe as antidiabetic therapy. There is no interaction while metformin and bitter gourd consumed once daily, but the interaction occurred in twice daily dose of bitter gourd. It can be lowering the blood glucose level of mice and induce hypoglycemia. Bitter gourd juice with concentration $77.58 \mathrm{~g} / \mathrm{mL}$ could reduce blood glucose level as metformin does. Histophatology observation shown the interaction affect the most in MBG group. Therefore, further research to find the real mechanism of bitter gourd compounds on diabetes mellitus pathophysiology is necessary.

\section{Acknowledgment}

We would like to thank the Head Department of Farmaka Tropis Laboratory, Faculty of Pharmacy, Universitas Mulawarman for giving us the free access to do this study and Head Department of Veterinary Pathology, Universitas Airlangga, (Mrs. Arimbi, drh., M.Kes, AP.), and also the vet and education staff from Department of Veterinary Pathology: Mr. Jumawan, S.Sos and Ms. Yayuk Istiti Rahayu for giving assistance in reading and preparing the histopathologically of organs in this study.

\section{References}

1. Tan SP, Kha TC, Parks SE, Roach PD. Bitter melon (Momordica charantia L.) bioactive composition and health benefits: a review. Food Rev Int. 2016; 32(2): 181-202.

2. Lo HY, Li CC, Ho TY, Hsiang CY. Identification of the bioactive and consensus peptide motif from Momordica charantia insulin receptor-binding protein. Food Chem . 2016; 204: 298-305. 
3. Foretz M, Guigas B, Bertrand L, Pollak M, Viollet B. Metformin: from mechanisms of action to therapies. Cell Metab. 2014; 20(6): 953-66.

4. Baxter K. Book Review: Stockley's Drug Interactions, 7th Edition. Ann. Pharmacother. 2006; 40(6): 1219.

5. Upadhyay J, Polyzos SA, Perakakis N, Thakkar B, Paschou SA, Katsiki N, et al. Pharmacotherapy of type 2 diabetes: An update. Metabolism. 2018; 78: 13-42.

6. Subramaniam S, Rosdi MHB, Kuppusamy UR. Customized cooking methods enhance antioxidant, antiglycemic, and insulin-like properties of Momordica charantia and Moringa oleifera. J Food Qual. 2017; 2017: 9561325. doi: 10.1155/2017/9561325.

7. Śmieszek A, Basińska K, Chrząstek K, Marycz K. In vitro and in vivo effects of metformin on osteopontin expression in mice adiposederived multipotent stromal cells and adipose tissue. 2015; 2015: 814896. doi: 10.1155/2015/814896.

8. Adak T, Samadi A, Ünal AZ, Sabuncuoğlu S. A reappraisal on metformin. Regul Toxicol Pharmacol. 2018; 92: 324-32.

9. Maniar K, Moideen A, Mittal A, Patil A, Chakrabarti A, Banerjee D. A story of metformin-butyrate synergism to control various pathological conditions as a consequence of gut microbiome modification: Genesis of a wonder drug ? Pharmacol Res. 2017; 117: 103-28.

10. Mohamed GA, Ibrahim SRM, Salah R, Dine E. Natural anti-obesity agents. Bull Fac Pharmacy. 2014; 52(2): 269-84.
11. Selvakumar G, Shathirapathiy G, Jainraj R, Yuvaraj Paul P. Immediate effect of bitter gourd, ash gourd, Knol-khol juices on blood sugar levels of patients with type 2 diabetes mellitus: a pilot study. J Tradit Complement Med. 2017; 7(4): 526-31.

12. Krawinkel MB, Ludwig C, Swai ME, Yang RY, Chun KP, Habicht $\mathrm{SD}$. Bitter gourd reduces elevated fasting plasma glucose levels in an intervention study among prediabetics in Tanzania. J Ethnopharmacol. 2018; 216: 1-7.

13. Zhang F, Lin L, Xie J. A mini-review of chemical and biological properties of polysaccharides from Momordica charantia. Int J Biol Macromol. 2016; 92(235): 246-53.

14. Ahmad N, Hasan N, Ahmad Z, Zishan M, Zohrameena S. Momordica charantia: for traditional uses and pharmacological actions. J Drug Deliv Ther. 2016; 6(2): 40-4.

15. Golub R, Tan J, Watanabe T, Brendolan A. Origin and immunological functions of spleen stromal cells. Trends Immunol. 2018; 39(6): 503-14.

16. Out M, Kooy A, Lehert P, Schalkwijk CA, Stehouwer CDA. Longterm treatment with metformin in type 2 diabetes and methylmalonic acid: Post hoc analysis of a randomized controlled 4.3 year trial. $\mathrm{J}$ Diabetes Complications. 2018; 32(2): 171-8.

17. National Toxicology Program [Internet]. Bethesda: US Department of Health and Human Services. Stomach, Glandular Stomach, Epithelium - Hyperplasia [updated: 2014 Nov 18; cited 2018 Dec 1]. Available from: https://ntp.niehs.nih.gov/nnl/alimentary/ glandular_stomach/epihyp/index.htm. 